\title{
Aus dem Vorwort zur 1. Auflage
}

Werkstattbuch - das klingt nach Arbeit, Werkzeug, Betrieb, Reparatur und eher nicht nach intellektuellem Sachbuch, Philosophie oder anderer anspruchsvoller Lektüre. Werkstattbuch - das ist ein Arbeitsbuch, wie ich es mir zu Beginn meiner Mediationstätigkeit vor jetzt bald 20 Jahren gewünscht hätte, ein Buch zum Nachschlagen, Konfrontieren, Überprüfen, Erinnern, Ausprobieren, Nachbessern, Vergleichen mit eigenen Erfahrungen. Ich hoffe sehr, dass es diesen Anspruch erfüllt und zur Diskussion und Praxisreflexion von Mediatorinnen und Mediatoren in den verschiedenen Feldern der Mediation, aber auch in der Lehre und Supervision beitragen kann.

Dieses Buch ist also ein Praxisbuch, obwohl es auch Theorie-, Methodik- und Reflexionsteile enthält. Es ist ein interdisziplinäres Buch. Viele andere Fachleute, vor allem Juristen aus der gemeinsamen Praxis, aus den vielen Ausbildungen und aus Supervisionen, haben insgeheim mit ihren Anregungen und ihrer Kritik daran mitgeschrieben. Unter anderem deshalb habe ich diesem Buch einen Erb-Mediationsfall zugrunde gelegt, weil in diesem Feld die Notwendigkeit der Zusammenarbeit von psychosozialen, juristischen und anderen Fachleuten sehr deutlich wird, und auch, weil Erb-Mediation im Grenzbereich von Familien- und Wirtschafts-Mediation liegt und damit auf interdisziplinäre Zusammenarbeit angewiesen ist.

Meine Hoffnung und meine eigene Erfahrung ist, dass sich die wesentlichen methodischen und technischen Mediationsbausteine auf die Baustellen der verschiedenen Mediationsfelder übertragen lassen - ganz sicher auch auf die Baustellen der eigenen alltäglichen Konfliktbewältigung im privaten und beruflichen, auch nachbarschaftlichen, wirtschaftlichen, sportlichen, politischen oder anderen Umfeld. Aus diesem Grund habe ich fast allen Kapiteln dieses Buches jeweils eine Übung für uns Mediatoren vorangestellt, damit wir nicht vergessen, dass Mediation nicht nur "den anderen" gut tut, sondern vor allem auch uns selbst, wenn wir uns darauf einlassen.

Ich danke allen, die am Entstehen und Korrigieren dieses Buches mitgearbeitet haben, besonders meiner stilistischen Lektorin, Frau Barbara Schermaier-Stöckl, und meinen anderen juristischen Freunden, besonders Herrn Walter J. Lehmann als meinem Beratungsanwalt in der Erbengemeinschaft des Verlags Schaller und Herrn Sebastian Strohmeier 
als dem Notar. Ich danke insbes. meinen drei wichtigsten amerikanischen Lehrern, Gary Friedman, Jack Himmelstein und dem verstorbenen John M. Haynes. Hier gilt immer noch das, was meine beiden Mitdenker in der Mediation und Mitverfasser des Buches „Familienmediation und Kinder", Heiner Krabbe und S. Cornelia Thomsen, und ich damals geschrieben haben: Es ist so viel gemeinsames Gedankengut inzwischen bei uns zusammengeflossen, dass kaum noch zu erkennen und $z$ unterscheiden ist, was von wem stammt und wer was wie weiterentwickelt hat. Deshalb ist die Literaturliste auch so kurz. Ich meine natürlich immer Mediatoren und Mediatorinnen - ich habe mich wegen der besseren Lesbarkeit der Texte für die kürzeste Version entschieden. Auch verwende ich lieber die lateinisch korrektere Form Medianden statt Medianten.

Zuletzt, aber nicht weniger intensiv, danke ich meinen beiden Verlagslektorinnen, Frau Dr. Stadlhofer-Wissinger und Frau Forner, für ihre inspirierende und geduldige Zusammenarbeit und ihre Unterstützung.

München, im September 2004

Hannelore Diez 